\title{
Mitigation of premature ovarian failure by over-expression of lentivirus vector-mediated Wilms tumor-suppressor gene
}

\author{
Juan Wang, Le Bo, Wendan Xu, Xuekai Li, Bin Jiang, Caiping Mao* \\ Reproductive Medicine Center, The First Affiliated Hospital of Soochow University, Suzhou, China \\ *For correspondence: Email: mp1161@163.com; Tel: 0086-512-67780720
}

Sent for review: 15 June 2018

Revised accepted: 27 August 2018

\begin{abstract}
Purpose: To investigate the effect of WT1(Wilms tumor-suppressor gene) overexpression on premature ovarian failure (POF)-mediated ovarian dysfunction.

Methods: Three mice groups were used: control group (untreated mice), POF group (mice sterilized by intravenous injection of cyclophosphamide and busulfan), and POF-LV(lentiviral vector) DGFP(green fluorescent protein) [WT1 group (POF mice given intra-ovarian microinjection of LVGGFPQWT1, a WT10overexpressing lentiviral vector, one week after sterilization). Real time-PCR was employed to analyze in vitro WT1 overexpression levels. Overall ovarian function was measured by hormonal assay, $H \& E$ staining, and immuno-histochemical techniques.

Results: Overexpression of WT1 in mice models of POF alleviated ovarian granulosa cell (GC) damage, increased ovary weight, and significantly increased follicular number $(p<0.05)$. Radioimmunoassays revealed reduction in plasma estradiol (E2) and follicle-stimulating hormone (FSH, $p<$ 0.05). However, results from immune-histochemical assays showed reduced Bax expression levels, and increased expression of Bcl-2 in WTI-overexpression mice, relative to POF mice.

Conclusion: Overexpression of WT1 may stimulate repair of ovarian tissue while improving endocrine function by inhibiting ovarian cell apoptosis signaling pathway in POF mice.
\end{abstract}

Keywords: Premature ovarian failure, Wilms tumor suppressor gene, Chemotherapy, Apoptosis, Estradiol

This is an Open Access article that uses a funding model which does not charge readers or their institutions for access and distributed under the terms of the Creative Commons Attribution License (http://creativecommons.org/licenses/by/4.0) and the Budapest Open Access Initiative (http://www.budapestopenaccessinitiative.org/read), which permit unrestricted use, distribution, and reproduction in any medium, provided the original work is properly credited.

Tropical Journal of Pharmaceutical Research is indexed by Science Citation Index (SciSearch), Scopus, International Pharmaceutical Abstract, Chemical Abstracts, Embase, Index Copernicus, EBSCO, African Index Medicus, JournalSeek, Journal Citation Reports/Science Edition, Directory of Open Access Journals (DOAJ), African Journal Online, Bioline International, Open-J-Gate and Pharmacy Abstracts

\section{INTRODUCTION}

Chemotherapy-induced POF and infertility are areas of immense interest to clinicians. This is due to rising incidence of malignant tumors and chemotherapy-induced POF, as well as increasing population of relatively young patients with malignant ovarian tumors $[1,2]$. Apart from POF and infertility, chemotherapy is accompanied by complications such as anorexia, amenorrhea, cardiovascular diseases and osteoporosis, all of which severely impact the life quality of young cancer survivors [3]. Fertilityenhancing treatments are available for POF patients. These include in vitro fertilization and hormonal therapy [4]. In contrast, there are limited options for patients with chemotherapyinduced POF. Thus, there is need for research 
aimed at developing treatment strategies for this group of POF patients. The WT1 gene is implicated in genito-urinary disorders $[5,6]$. It is an important inhibitor of cellular apoptosis in male germline cells, and in the embryological development of the kidney [7]. It has been demonstrated that granulosa cell (GC) survival is dependent on WT1 activation especially at the initial stages of development of the follicles [8]. Moreover, it is involved in the control of the effectors of the apoptotic cascade [8]. Nevertheless, not much is known from direct in vivo investigations on the apoptosis-promoting or apoptosis-inhibitory effects of WTI protein, and its effect on the ovary is poorly understood.

In gene therapy, DNA-based RNAi can be stably introduced for once-treatment of shRNA genes from viral vectors [9]. The transfer of genes by lentivirus vectors is more beneficial when compared with gene transfer by other carriers. Cells can be infected irrespective of whether they are fully differentiated or undergoing active division. Besides, lentiviruses are easily incorporated into host genome, and they do not elaborate gene products $[10,11]$. Several studies have shown that POF is implicated in granulocyte apoptosis. The hypothesis that formed the plank of the present investigation was that lentivirus-mediated WT1 overexpression in GCs might induce transcription of genes necessary for conferring an advantage in cell survival. Thus, the effect of WTI overexpression on endocrine function and follicular development in POF mice was studied. The study was also carried out to investigate the link between the effects of WTI overexpression and the Bax and bcl-2 genes, which are integral components of cell life and death [12].

\section{EXPERIMENTAL}

\section{Animals and chemicals}

Two month-old female C57BL/6 mice were used in this study. They were purchased from JOINN Laboratories (Suzhou). This research was approved by the Animal Ethical Committee of The First Affiliated Hospital of Soochow University, according to "Principles of Laboratory Animal Care" (NIH publication no. 85-23, revised 1985) [13], the approval number is No.32-87, 2017. All reagents used in this study were products of either Sigma Chemical Co. (St Louis, $\mathrm{MO}$, USA, or Sigma-Aldrich).

\section{Granulosa cells (GCs)}

Following cervical dislocation, mice ovaries were removed under sterile conditions. After washing thrice in PBS, the ovaries were placed in DMEM/F12(Dulbecco's Modified Eagle Media: Nutrient Mixture F-12) with $5 \%$ fetal bovine serum (FBS) in a cell incubator for $15 \mathrm{~min}$. Ovarian follicle tissue was transferred by repeated fine needle puncture, into the DMEM culture medium containing $0.1 \%$ hyaluronidase and digested for 2 min. Ovarian debris was removed by filtration through a 200 mesh sieve. After one rinse, the cells were maintained on DMEM/F12 culture medium containing $10 \%$ fetal bovine serum, $100 \mu \mathrm{g} / \mathrm{ml}$ streptomycin sulfate and $100 \mathrm{lU} / \mathrm{ml}$ penicillin, overnight for adhesion.

\section{Mice groups and treatments}

The study used 3 mice groups, each containing 6 mice: control group (untreated mice), POF group (age-matched mice sterilized with intraperitoneal injection of $120 \mathrm{mg}$ cyclophosphamide $/ \mathrm{kg}$ body weight), and POF-LV?GFP?WT1 group (POF mice given intra-ovarian microinjection of $5 \mu$ of LV?GFP?WT1 at a dose of $2 \times 10^{8}$ titer units/ml/ovary, and $30 \mathrm{mg}$ busulfan $/ \mathrm{kg}$ body weight, one week after sterilization). The cyclophosphamide and busulfan used were products of Sigma-Aldrich. Prior to injection, they were re-suspended in a 1:2 (v:v) mixture of DMSO(dimethyl sulfoxide) and PBS (phosphate buffer saline).

\section{Design and construction of lentivirus vector}

WT1-targeting oligonucleotides [WT1(NM_144783)] are shown in Figure 1. The lentivirus encoding the complete WT1 open reading frame (LV国GFP?WT1) and negative control virus were obtained from GeneChem Biotechnology (Shanghai, China). The concentration of the stock lentiviral suspension was $5 \times 10^{8}$ titer units (TU)/ml.

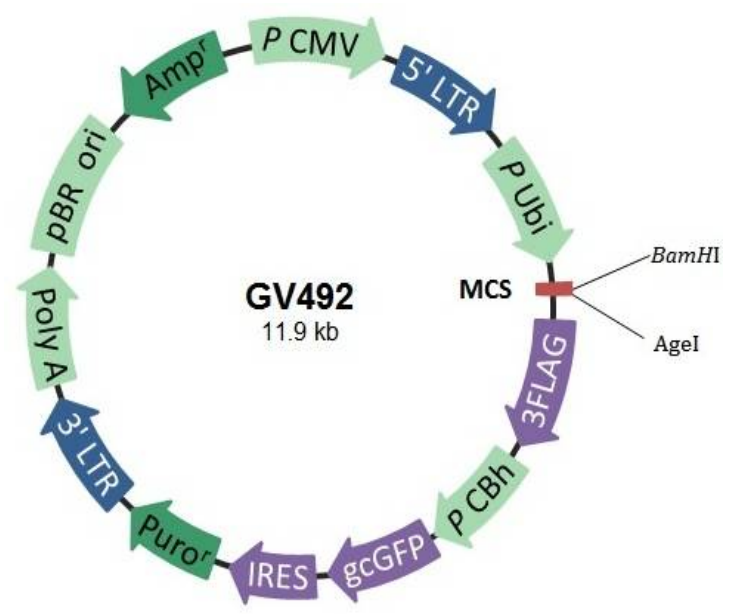

Figure 1: WT1-overexpressing lentiviral vector map

Trop J Pharm Res, September 2018; 17(9): 1746 


\section{Injection of lentivirus}

The mice ovaries were injected with lentivirus via the subscapular approach by means of a 10- $\mu$ l syringe (Nanofil Syringe, WPI). Slow injections were carried out, with needles kept in place for 5 min. Each ovary had a total injection of $5 \mu \mathrm{l}$, and each mouse received a total of $5 \mu$ l of lentivirus.

\section{Hormone assays}

On day 42 after cyclophosphamide and busulfan treatment, whole blood samples $(1.0$ - $1.2 \mathrm{ml})$ were drawn (into anticoagulated containers) under anesthesia through the abdominal aorta. Following a $3-\mathrm{h}$ incubation at $37{ }^{\circ} \mathrm{C}$, plasma samples were obtained by centrifugation, and used for the assays of FSH and E2 (as indicators of ovarian senescence) with radioimmunoassay (RIA) techniques at the Beijing Sino-uk Institute of Biological Technology.

\section{Ovarian morphologies and follicle enumeration}

Ovarian tissue samples were fixed in paraformaldehyde and paraffinized after sequential dehydration in ethanol. Tissue sections were subjected to $\mathrm{H}$ \& $\mathrm{E}$ staining in line with standard procedures, and the various follicular forms were enumerated under the light microscope.

\section{Immunohistochemistry}

Para-formaldehyde-fixed and paraffinized ovarian tissues were sectioned and microwaved after rinsing in phosphate buffer. The sections were subsequently incubated for $45 \mathrm{~min}$ at $37^{\circ} \mathrm{C}$ with anti-WT1 (ab89901, Abcam), anti-Bax (2772T, CST) and 3498T (anti-Bcl-2). Following incubation with secondary antibody, visualization was enhanced by addition of $A B C$ chromogenic reagent. The negative control was PBS. Using a light Olympus microscope, 5 views per section were assessed and used for quantification of immune-reactive cells.

\section{Real-time PCR}

In the determination of relative mRNA expression levels, mice ovarian tissue or granulosa cells were lysed with TRIzol Reagent (Beyotime
Institute of Biotechnology, Shanghai, China). Total RNA was obtained as per manufacturer's protocol, and reverse-transcribed to cDNA. Then, qRT-PCR was done on Applied Biosystems 7500 with specific SYBR Premix Ex TaqTM, (primer sequences are depicted in Table 1). The qPCR and the $2^{-\triangle \triangle C T}$ methods were used for determination of relative gene expressions. Mean fold changes were obtained from three independent experiments.

\section{Statistical analysis}

Each experiment was performed at least three times, and results are presented as mean \pm standard error of the mean (SEM). The data were analyzed using GraphPad Prism software (version 5.0; GraphPad Software, Inc., La Jolla, CA, USA). Where applicable, differences were evaluated by Student's t? test. Values of $p<0.05$ were considered statistically significant.

\section{RESULTS}

\section{Effect of LV-GFP-WT1 infection on basal WT1 transcript levels in GCs}

Granular cells (GCs) are not susceptible to infection. Thus, to achieve more than $80 \%$ infection efficiency, the $\mathrm{MOI}$ should be about 100. A $90 \%$ lentiviral infection efficiency was successfully effected in GCs by LV-GFP-WT1 treatment, as confirmed by quantification of GFP expression $72 \mathrm{~h}$ after transfection (Figure $2 \mathrm{~A}$ ). In addition, the negative control (NC) and blank control groups were established. There were significant increases in endogenous WT1 mRNA expressions in the WT1-overexpression group 72 $\mathrm{h}$ post-infection, when compared to the control group and NC group ( $p<0.05$, Figure $2 \mathrm{~B})$.

\section{Identification of mouse ovarian tissue transfected with LV-GFP-WT1}

Fluorescence expression was observed under an inverted fluorescence microscope and the results are shown in Figure 3. Except for the control group, the POF group and the LV-GFP-WT1 group expressed green fluorescence. Ovarian granulosa cells and ovarian epithelial cells were expressed in green fluorescence.

Table 1: Gene primer sequences used

\begin{tabular}{llc}
\hline Primer & Primer( (5'-3') & Product size (bp) \\
\hline WT1 & FP: AGCACGGTCACTTTCGACG & 85 \\
& RP:GTTTGAAGGAATGGTTGGGGAA & \\
$\beta$-actin & FP: GAGACCTTCAACACCCCAGC & 263 \\
\hline
\end{tabular}


A

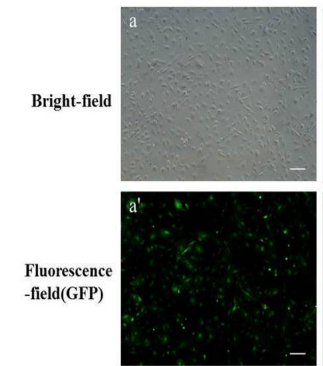

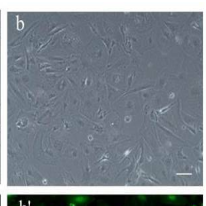
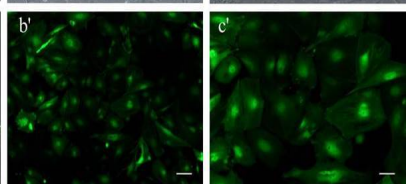

B

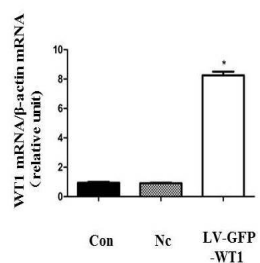

WT1

Figure 2: Enhancement of WTI expression in GCs by lentiviral-mediated RNAi. A: C cells infected with lentivirus at an MOI of 100. (scale bars $=400,200$ and $100 \mu \mathrm{m}$ for $\mathrm{a}, \mathrm{a}^{\prime} ; \mathrm{b}, \mathrm{b}^{\prime}$; and $\mathrm{c}, \mathrm{c}^{\prime}$, respectively. B: Relative expression of WT1 mRNA in GCs; ${ }^{*} p<0.05$, vs control and negative control groups. (Con $=$ control, $\mathrm{Nc}=$ negative control)

POF
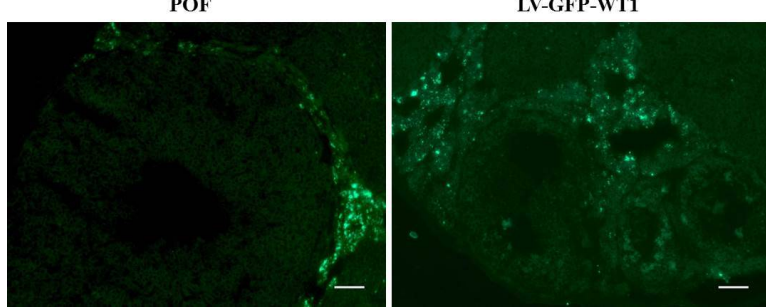

Figure 3: Fluorescent images of mouse ovarian tissue after transfection. Scale bar: $200 \mu \mathrm{m}$

\section{WT1 was significantly overexpressed in POF- LV-GFP-WT1 groups}

Immuno-histochemical staining was performed on ovarian tissues to localize WT1. As shown in Figure 4 A, WT1 was expressed specifically in the nucleus of GCs, a finding that is consistent with the results obtained by previous investigators. The POF group had significantlyattenuated WT1 levels, relative to the control and POF-LV-GFP-WT1 groups (Figure $4 \mathrm{~B}, p<0.05$ ).

\section{WT1 overexpression increased normal follicular cells, endocrine function, and ovarian weight in POF mice}

Mice ovaries in the control group contained a variety of mature and immature follicles at every developmental stage, as revealed by $H$ \& $E$ staining (Figure $5 \mathrm{~A}$ ). In contrast, ovaries of POF mice had fewer follicles at every developmental stage, and consisted mainly of interstitial cells. The ovaries of POF mice were generally smaller in size than those from control group. However,
WTI overexpression in mice with POF was able to significantly reverse ovarian pathology and alleviate the degree of injury in ovarian GCs (Figure $5 \mathrm{~A}$ ). Cell-counting of normal follicles revealed an absolute reduction in follicles in mice with POF ( $p<0.05$; Figure $5 \mathrm{~B}$ ). The number of follicles in the POF-LV-GFP-WT1 group was higher than that in the POF group, but cell counts of normal follicles differed significantly between the POF-LV-GFP-WT1 group and the control group $(p<0.05)$.
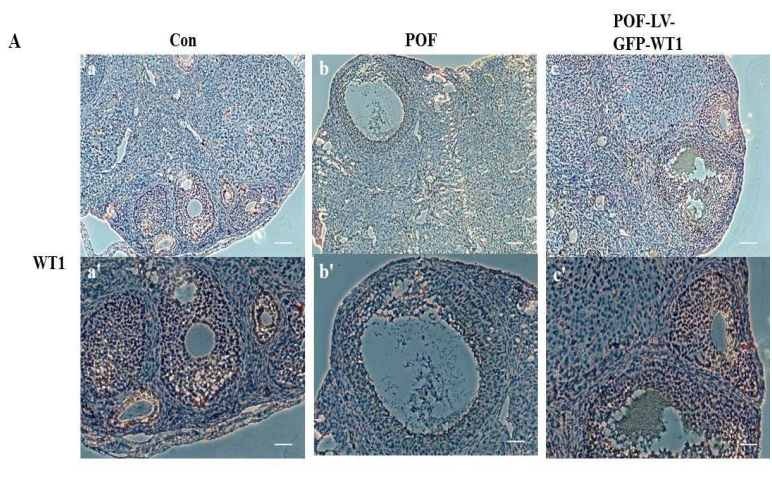

B

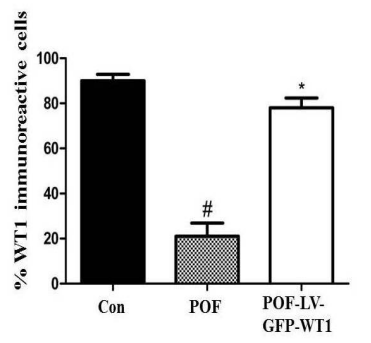

Figure 4: Immuno-histochemical detection of WT1 protein in the ovary. A: Expression of WT1 in ovarian granulosa cells (scale bars for a, b, c and a', b', and c' are 400 and $200 \mu \mathrm{m}$, respectively. B: Quantitation of WT1 immuno-reactive cells. WT1 was significantly increased in POF-LV-GFP-WT1 group, when compared with the expression in POF group; ${ }^{*} p<$ 0.05 , compared to POF group; \#p $<0.05$, relative to control

Ovary weight of mice in the POF and control groups differed significantly (Figure $5 \mathrm{C}$ ). However, the weight of ovarian tissue did not significantly differ between the POF and POFLV-GFP-WT1 mice groups ( $p>0.05)$. In the POF group, there were reduced blood levels of E2 but elevated levels of $\mathrm{FSH}$, relative to the control group. Although E2 and FSH levels were also different between the POF-LV-GFP-WT1and control groups, mice in the POF-LV-GFP-WT1 group had elevated plasma E2 and reduced FSH levels, relative to the POF group (Figure $5 \mathrm{D}$ and E).

\section{WT1 overexpression in GCs affected Bax and bcl-2 protein levels}

The proportion of Bax-immunoreactive cells 
A

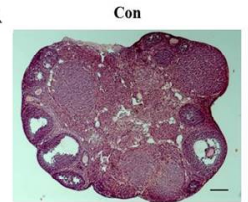

B
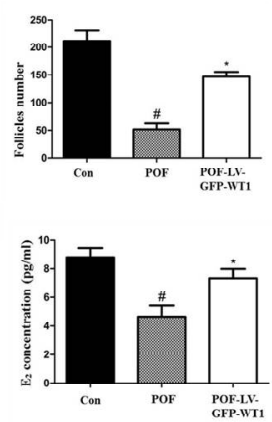

POF-LV-GFP-WT1

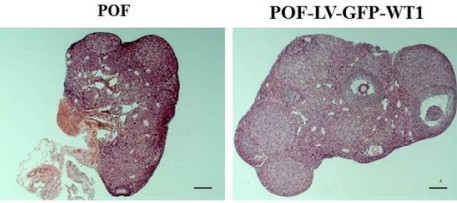

C
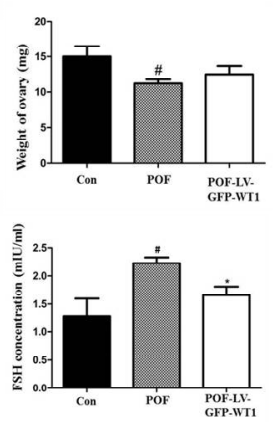

Figure 5: Effect of WT1 overexpression on POF. A: WTI overexpression enhanced the population of normal follicles and decreased atretic follicles in POFinduced ovarian tissue pathology. B: Follicular number in POF-LV-GFP-WT1 group was significantly increased, relative to POF group. C: Ovarian tissue weight did not significantly differ between the POF-LVGFP-WT1 and the POF group. D: $E_{2}$ content of plasma was higher in POF-LV-GFP-WT1 group, relative to POF group. $\mathrm{E}$ : Plasma $\mathrm{FSH}$ were lower in the POF-LV-GFP-WT1 group, relative to the POF group. Values are mean $\pm \mathrm{SEM}$; ${ }^{*} p<0.05$, compared to control and POF groups; \#p $<0.05$, compared to control

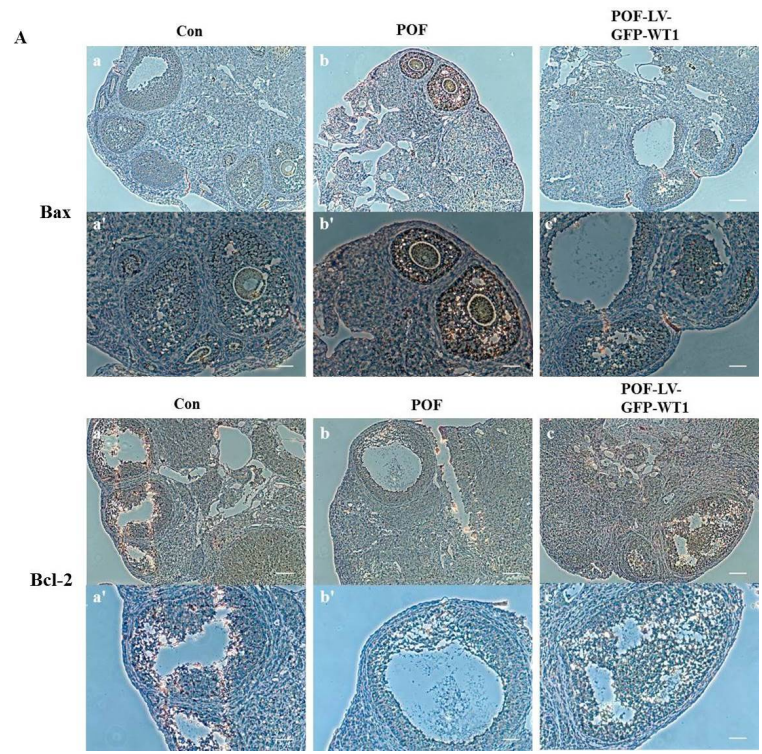

Figure 6: Immuno-histochemical localization of Bax and bcl-2 proteins in the ovary. A: Bax and Bcl-2 were expressed mainly in ovarian granulosa cells (scale bars: 400 and $200 \mu \mathrm{m}$ for $a, b, c$; and $a^{\prime} b^{\prime} c^{\prime}$, respectively). B: Quantitation of $\mathrm{Bax}$ and bcl-2 immuno-reactive cells. Bax was significantly decreased in POF-LV-GFP-WT1 group, when compared with Bax expression in POF group. Bcl-2 was significantly increased in POF-LV-GFP-WT1 group, relative to its expression in POF group; ${ }^{*} p<$ 0.05 , relative to POF and control groups; $\# p<0.05$, compared to control and POF groups

(percent of total cells) in POF-LV-GFP-WT1 group was significantly increased, when compared with the control group, but was lower than the value obtained in the POF group $(p<$ 0.05; Figure $6 \mathrm{~B}$ ). However, the percentage of bcl-2-immunoreactive cells in POF-LV-GFP-WT1 group was lower than that in the control group, but significantly higher than corresponding levels in the POF group $(p<0.05)$.

\section{DISCUSSION}

Premenopausal women subjected to chemotherapy treatment are at high risk of POF. Currently, there are some treatments for POF patients. However, there is still a need for improvement in the treatment strategies. The results obtained in the present study revealed that ovarian dysfunction in chemotherapyinduced ovarian injury could be mitigated by the overexpression of WT1 induced by intra-ovarian microinjection of lentiviral vectors in mouse ovaries.

Alkylating agents (e.g. cyclophosphamide and busulfan) are considered more gonadotoxic than other agents. It has been reported that about 4 out of every 10 women subjected to alkylating agent therapy experienced ovarian failure, in contrast to those who received alkaloid or platinum-based therapy [14]. Alkylating agents exert their deleterious effects on cells through the induction of DNA cross-links [15]. Cross-links in DNA influence mitochondrial dynamics and activate the process of apoptosis [16]. Studies have shown that cyclophosphamide increases the levels of pro-apoptotic protein Bax [16]. Therefore, cyclophosphamide significantly reduces GCs, compromises the development of follicles and reduces the population of oocytes $[17,18]$. Agents with alkylating properties impair follicular growth and decrease the populations of all types of follicles [19].

Estrogen is secreted mainly by ovarian GCs. Chemotherapeutic drugs reduce the level of estradiol due to damage to GCs. The results of this study are consistent with the finding that cyclophosphamide induced apoptosis in GCs, resulting in the reduction of various types of follicles and impairment of ovarian endocrine function [20].

The fates of follicular cells are dictated by the relative expressions of tumor suppressors, survival factors and apoptotic proteins [21]. It is known that WT1 is one of the zinc finger transcription that are strongly expressed in GCs [21]. Studies by Kreidberg et al established for 
the first time that WT1 inhibits apoptosis associated with urogenital tract development [22]. Studies on the effect of WT1 on GC programmed cell death are carried out in the absence of serum so as to provoke spontaneous apoptosis [23]. Both Bax and bcl-2 proteins are primarily involved in the regulation of the secretion of pro-apoptotic factors in several stages of the apoptotic cascade [4]. These proteins are in turn regulated by WT1, which also modulates several other pro-apoptotic genes in non-gonadal cells $[16,24,25]$.

In the present study, LV-GFP-WT1 was applied to study the role of significantly overexpressed WT1 at the levels of gene transcription and protein expression. It was found that WT1 possesses the potential to repair ovarian injury and augment ovarian function. The POF mice which were modified to overexpress WT1 had a diminished level of Bax immune-reactive cells and elevated percentage of Bcl-2 immunoreactive cells, when compared to POF mice that did not have WT1 overexpression. Thus, WT1 may affect apoptosis by influencing the expressions of apoptotic signaling pathway factors, particularly Bax and Bcl-2. Although the exact cellular processes involved are yet to be delineated, the finding that WT1 influences the expression of factors in apoptotic signaling pathways is a novel information on the control of apoptosis in follicular development. Thus, the present study has identified a novel target molecule for the development of a new therapy for chemotherapy-induced POF.

\section{Study limitations}

The procedures used for delivering the lentivirus vectors into the ovaries involved tedious operations which may affect the safety of the vectors themselves. Further studies are necessary to deepen the current understanding of how WT1 influences the expression of factors involved in the apoptotic cascade.

\section{CONCLUSION}

It can be reasonably inferred that overexpression of WTI gene enhances recovery of ovarian tissue and stimulates release of estrogen via the activation of factors involved in the apoptotic signaling pathways that exist in ovarian tissue of POF mice models. Therefore, WT1 may serve as a potential novel marker for POF induced by cyclophosphamide and a new target for treatment. Future studies are required to investigate the specific mechanism underlying the effect of POF on WT1.

\section{DECLARATIONS}

\section{Acknowledgement}

This study was funded by National Natural Science Foundation (81370719 and 81671535), Jiangsu Science Foundation (BE2015642), Jiangsu Key Discipline of Human Assisted Reproduction Medicine Foundation (FXK201749), and Jiangsu Provincial Medical Talent of the Project of Invigorating Healthcare through Science, Technology and Education (ZDRCA2016044).

\section{Conflict of interest}

The author(s) declared no potential conflict of interest with respect to the research, authorship, and/or publication of this article.

\section{Contribution of authors}

We declare that this work was done by the author(s) named in this article and all liabilities pertaining to claims relating to the content of this article will be borne by the authors, all authors read and approved the manuscript for publication. Juan Wang contributed to the design of the study, conducting the study, data collection, data analysis, and writing the manuscript. Le Bo contributed to the design of the study and data analysis. Wendan Xu contributed to data interpretation. Xuekai Li and Bin Jiang contributed to data collection. Caiping Mao contributed to the design of the study and revising the manuscript.

\section{REFERENCES}

1. Swerdlow AJ, Cooke R, Bates A, Cunningham D, Falk SJ, Gilson D, Hancock BW, Harris SJ, Horwich A, Hoskin PJ, et al. Risk of premature menopause after treatment for Hodgkin's lymphoma. J Natl Cancer Inst 2014; 106(9): 1-12.

2. Wan J, Gai Y, Li G, Tao Z, Zhang Z. Incidence of chemotherapy- and chemoradiotherapy-induced amenorrhea in premenopausal women with stage II/III colorectal cancer. Clin Colorectal Cancer 2015; 14(1): 31-34.

3. Benetti-Pinto CL, de Almeida DM, Makuch MY. Quality of life in women with premature ovarian failure. Gynecol Endocrinol 2011; 27(9): 645-649.

4. Vujovic S, Ivovic M, Tancic-Gajic M, Marina L, Barac $M$, Arizanovic Z, Nenezic A, Lvanisević M, Micic J, Sajic S, et al. Premature ovarian failure. Srp Arh Celok Lek 2012; 140(11): 806-811. 
5. Hastie ND. Wilms' tumour 1 (WT1) in development, homeostasis and disease. Development 2017; 144(16): 2862-2872.

6. Hashimoto $H$, Zhang $X$, Zheng $Y$, Wilson GG, Cheng $X$. Denys-Drash syndrome associated WT1 glutamine 369 mutants have altered sequence-preferences and altered responses to epigenetic modifications. Nucleic Acids Res 2016; 44(21): 10165-10176.

7. Rao MK, Pham J, Imam JS, Maclean JA, Murali D, Furuta $Y$, Sinha-Hikim AP, Wilkinson MF. Tissuespecific RNAi reveals that WT1 expression in nurse cells controls germ cell survival and spermatogenesis. Genes Dev 2006; 20(2): 147-152.

8. Park M, Choi Y, Choi H, Roh J. Wilms' tumor suppressor gene (WT1) suppresses apoptosis by transcriptionally downregulating $B A X$ expression in immature rat granulosa cells. J Ovarian Res 2014; 7: 118.

9. Aagaard L, Rossi JJ. RNAi therapeutics: principles, prospects and challenges. Adv Drug Deliv Rev 2007; 59(2): 75-86.

10. Romano G. Current development of lentiviral-mediated gene transfer. Drug News Perspect 2005; 18(2): 128134.

11. Song $L P$, Li YP, Qiu SD, Wang N. Recombinant lentivirus-mediated gene transfer of NT4-p53(N15)-Ant inhibits the growth of hepatocellular carcinoma cells in vitro. Chin J Oncol 2010; 32(1): 10-6.

12. Williams GT, Smith CA. Molecular regulation of apoptosis: genetic controls on cell death. Cell 1993; 74(5): 777-779.

13. World Health Organization. Principles of laboratory animal care. WHO Chron 1985; 39: 51-56.

14. Meirow D. Reproduction post-chemotherapy in young cancer patients. Mol Cell Endocrinol 2000; 169(1-2): 123-131.

15. Helleday T, Petermann E, Lundin C, Hodgson B, Sharma $R A$. DNA repair pathways as targets for cancer therapy. Nat Rev Cancer 2008; 8(3): 193-204.
16. Zhao $X J$, Huang $Y H, Y u Y C$, Xin $X Y$. GnRH antagonist cetrorelix inhibits mitochondria-dependent apoptosis triggered by chemotherapy in granulosa cells of rats. Gynecol Oncol 2010; 118(1): 69-75.

17. Desmeules P, Devine PJ. Characterizing the ovotoxicity of cyclophosphamide metabolites on cultured mouse ovaries. Toxicol Sci 2006; 90(2): 500-509.

18. Matzuk MM, Burns KH, Viveiros MM, Eppig JJ. Intercellular communication in the mammalian ovary: oocytes carry the conversation. Science 2002; 296(5576): 2178-2180.

19. Rosendahl M, Andersen CY, la CFN, Juul A, Løssl K, Andersen AN. Dynamics and mechanisms of chemotherapy-induced ovarian follicular depletion in women of fertile age. Fertil Steril 2010; 94(1): 156-166.

20. Lopez SG, Luderer U. Effects of cyclophosphamide and buthionine sulfoximine on ovarian glutathione and apoptosis. Free Radic Biol Med 2004; 36(11): 13661377.

21. Asselin E, Xiao CW, Wang YF, Tsang BK. Mammalian follicular development and atresia: role of apoptosis. Biol Signals Recept 2000; 9(2): 87-95.

22. Kreidberg JA, Sariola H, Loring JM, Maeda M, Pelletier J, Housman $D$, Jaenisch $R$. WT-1 is required for early kidney development. Cell 1993; 74(4): 679-691.

23. Tilly JL, Billig $H$, Kowalski Kl, Hsueh AJ. Epidermal growth factor and basic fibroblast growth factor suppress the spontaneous onset of apoptosis in cultured rat ovarian granulosa cells and follicles by a tyrosine kinase-dependent mechanism. Mol Endocrinol 1992; 6(11): 1942-1950.

24. Garner TP, Lopez A, Reyna DE, Spitz AZ, Gavathiotis E. Progress in targeting the BCL-2 family of proteins. Curr Opin Chem Biol 2017; 39: 133-142.

25. An $P$, Yin $Y$, Fan A, Tan W, Gao F, Ma K, Tang B, Zhang $X$, Li Z. effects of the WT1 gene on apoptosis and development-related gene expression in porcine kidney fibroblasts and swine testis cells. Mol Reprod Dev 2013; 80(5): 414-425. 